\title{
Presença de hidrocarbonetos policíclicos aromáticos em produtos alimentícios e a sua relação com o método de cocção e a natureza do alimento
}

\author{
The presence of polycyclic aromatic hydrocarbons in food products and their \\ relationship with the cooking method and nature of the food
}

\author{
Allane Patricia Santos da Paz ${ }^{1 \star}$, Ellen Cristina Pimenta Nascimento', Hélcio Cassemiro Marcondes², \\ Márcia Cristina Freitas da Silva ${ }^{3}$, Moises Hamoy ${ }^{4}$, Vanessa Jóia de Mello ${ }^{4}$ \\ 1 Universidade Federal do Pará, Instituto de Ciências da Saúde, Faculdade de Nutrição, Belém/PA - Brasil \\ 2 Universidade Federal de Ouro Preto, Instituto de Ciências Exatas e Biológicas, Ouro Preto/MG - Brasil \\ ${ }^{3}$ Universidade Federal do Pará, Instituto de Ciências Biológicas, Belém/PA - Brasil \\ ${ }^{4}$ Universidade Federal do Pará, Instituto de Ciências Biológicas, Laboratório de Farmacologia e Toxicologia de Produtos Naturais, Belém/PA - Brasil
}

\section{*Corresponding Author}

Allane Patricia Santos da Paz, Universidade Federal do Pará (UFPA), Instituto de Ciências da Saúde, Faculdade de Nutrição, Rua Augusto Corrêa, 1, Guamá, CEP: 66075-110,Belém/PA - Brasil, e-mail: allanepatricia.paz@gmail.com

Cite as: The presence of polycyclic aromatic hydrocarbons in food products and their relationship with the cooking method and nature of the food. Braz. J. Food Technol., v. 20, e2016102, 2017.

Received: Aug. 26, 2016; Accepted: June 07, 2017

\section{Resumo}

Hidrocarbonetos Policíclicos Aromáticos (HPA) podem estar associados à carcinogênese em humanos. Tais compostos penetram no organismo pelo trato gastrointestinal, o que faz da dieta uma importante via de contaminação. O objetivo desta revisão é analisar a relação entre a formação/ingestão desses compostos e a alimentação. Foi encontrada associação direta do método de cocção empregado com o aumento dos níveis de HPA nos alimentos e a formação de novos compostos. A fonte térmica aplicada, a composição do alimento, o tipo de óleo utilizado, especialmente nos processos de fritura, bem como o tipo de tratamento empregado ao alimento antes da cocção, são fatores que influenciam o teor de HPA no produto final. A legislação brasileira é pouco abrangente em relação a esses compostos e a necessidade de ampliação das normas nacionais se torna ainda mais evidente quando este tema é visto como uma questão de Segurança de Alimentos.

Palavras-chave: Hidrocarbonetos Policíclicos Aromáticos; Alimentação; Nutrição; Câncer.

\section{Abstract}

Polycyclic Aromatic Hydrocarbons (PAH) can be associated with carcinogenesis in humans. These elements enter the body via the gastrointestinal tract, making the diet an important contamination route. The objective of this review was to analyze the comprehensive relationship between the formation/ingestion of these compounds and the diet. A significant association was found between the cooking method and the increase in PAH levels in foods and the formation of new compounds. The thermal source applied, food composition, type of oil, especially in the frying processes, as well as the type of treatment used before cooking, influenced the PAH content in the final product. Brazilian legislation for these compounds is not comprehensive and the need to expand national standards becomes even more evident when this issue is seen as a matter of Food Safety.

Keywords: Polycyclic Aromatic Hydrocarbons; Feeding; Nutrition; Cancer.

\section{Introdução}

A industrialização da sociedade moderna tem originado uma variedade de poluentes que se relacionam intimamente com o aparecimento de diversos tipos de câncer (COYLE,

2004; ZHANG et al., 2009; XIA et al., 2010) e, dentre estes contaminantes, estão os Hidrocarbonetos Policíclicos Aromáticos (HPA). Muitos destes compostos apresentam 
Presença de hidrocarbonetos policíclicos aromáticos em produtos alimentícios e a sua relação com o método de cocção e a natureza do alimento Paz, A. P. S. et al.

potencial carcinogênico, sendo associados diretamente à carcinogênese em humanos (SHIMADA, 2006; IARC, 2010; TARANTINI et al., 2011). Nesta classe, estão incluídos mais de cem compostos químicos, que podem penetrar no organismo através do trato gastrintestinal, dos pulmões e/ou serem absorvidos pela pele (FAN et al., 2006; ZHANG et al., 2010; NACCARI et al., 2011). Contudo, 16 HPA são considerados prioritários pela Agência de Proteção Ambiental Americana (US EPA, 1998) e classificados, segundo a Agência Internacional para Pesquisa do Câncer (IARC, 2013), de acordo com suas propriedades carcinogênicas e mutagênicas (Tabela 1).

Desmembrando as fontes geradoras de HPA, às quais a sociedade se encontra mais exposta, destacam-se: a queima de combustíveis (ZHANG; TAO, 2009), o processo de defumação e cocção de carnes (XIA et al., 2010; FARHADIAN et al., 2010; ROSEIRO et al., 2011), as frituras sob temperatura extremamente elevada (WRETLING, et al., 2010; HARVEY, 1982), a fumaça de tabaco (HARVEY, 1982; CHUNG et al., 2011) e o consumo de óleos vegetais (CAMARGO et al., 2012).

Os diversos tipos de contaminantes encontrados em alimentos podem ser de caráter físico, químico ou biológico, sendo comumente resultado de determinados fatores, como poluição ambiental ou modo de cocção (BALBANI; BUTUGA, 2001; SPISSO et al., 2009). Os HPA são poluentes químicos que se enquadram nestas vias clássicas de contaminação.

Sob esta ótica, os HPA são compostos que merecem atenção no campo da Segurança de Alimentos, pois a crescente preocupação com a presença de contaminantes em alimentos faz, deste, um tema de grande interesse não apenas para os pesquisadores da área, mas também para a população de modo geral, uma vez que a busca por qualidade de vida vem moldando consumidores cada vez mais conscientes do direito de adquirir produtos seguros à saúde (BOBEDA et al., 2013).

Com base nisso, este trabalho foi desenvolvido com o objetivo de analisar a estreita relação entre os contaminantes em questão e a dieta humana, levando em conta estudos desenvolvidos em nível nacional e internacional, que investigaram a presença de contaminação por HPA em diversos tipos de alimentos e situações. Foram utilizadas, no desenvolvimento desta revisão bibliográfica, publicações que associassem o termo "HPA" aos seguintes temas: metabolismo; carcinogênese; composição dos alimentos; modo de cocção; condições de armazenamento e conservação; contaminação ambiental.

\section{Aspectos gerais da formação dos HPA}

HPA são compostos de baixa hidrossolubilidade, mostrando-se geralmente muito lipossolúveis; são produzidos por combustão incompleta ou pirólise de matéria orgânica (HOWARD; FAZIO, 1980; DANYI et al., 2009; TFOUNI et al., 2009). Sua síntese é influenciada pelos seguintes fatores: tipo de biomassa presente, pressão, quantidade de oxigênio disponível e, principalmente, de calor (CONDE et al., 2004).

A formação pirolítica é bastante complexa e variável e seu mecanismo envolve temperaturas elevadas, em que compostos orgânicos são convertidos em moléculas pequenas não estáveis (LOPES; ANDRADE, 1996; CARUSO; ALABURDA, 2008). Na pirossíntese, essas e outras

Tabela 1. HPA considerados prioritários pela Agência de Proteção Ambiental Americana (US EPA, 1998) e sua classificação segundo a Agência Internacional para Pesquisa do Câncer (IARC, 2013).

\begin{tabular}{|c|c|c|c|}
\hline Compostos & Genotoxidade & Classificação IARC & N. ${ }^{\circ}$ de anéis \\
\hline Naftaleno & Positivo & Possivelmente carcinogênico & 2 \\
\hline Acenafteno & Questionável & Ainda não avaliado & 3 \\
\hline Acenaftileno & Questionável & Ainda não avaliado & 3 \\
\hline Antraceno & Negativo & Não classificado & 3 \\
\hline Fluoreno & Negativo & Não classificado & 3 \\
\hline Fenantreno & Questionável & Não classificado & 3 \\
\hline Fluoranteno & Positivo & Não classificado & 4 \\
\hline Pireno & Questionável & Não classificado & 4 \\
\hline Benzo(a)antraceno & Positivo & Possivelmente carcinogênico & 4 \\
\hline Criseno & Positivo & Possivelmente carcinogênico & 4 \\
\hline Benzo(b)fluoranteno & Positivo & Possivelmente carcinogênico & 5 \\
\hline Benzo(k)fluoranteno & Positivo & Possivelmente carcinogênico & 5 \\
\hline Dibenzo(a,h)antraceno & Positivo & Provavelmente carcinogênico & 5 \\
\hline Benzo(a) pireno & Positivo & Carcinogênico & 5 \\
\hline Indeno(1,2,3-cd)pireno & Positivo & Possivelmente carcinogênico & 6 \\
\hline Benzo(g,h,i)perileno & Positivo & Não classificado & 6 \\
\hline
\end{tabular}

Fonte: Adaptado de Singh et al. (2016). 
Presença de hidrocarbonetos policíclicos aromáticos em produtos alimentícios e a sua relação com o método de cocção e a natureza do alimento Paz, A. P. S. et al.

sustâncias são recombinadas e produzem moléculas de HPA maiores e mais estáveis (BETTIN; FRANCO, 2005).

Os HPA são divididos em compostos de maior ou menor massa molecular: os mais leves têm de dois a três anéis aromáticos, enquanto os mais pesados possuem quatro ou mais anéis (SIMONEIT, 2002). Os compostos mais pesados, além de mais estáveis, possuem maior caráter lipofílico, característica que facilita sua absorção pelo corpo (REY-SALGUEIRO et al., 2009). Desta forma, de modo geral, são considerados mais preocupantes quanto à genotoxidade, como visto na Tabela 1 .

\section{Alimentos e HPA}

Em indivíduos não fumantes, a dieta é a principal fonte de contaminação por HPA (LODOVICl et al., 1995; PHILLIPS, 1999; FALCÓ et al., 2003), correspondendo a aproximadamente $70 \%$ da exposição total (GILBERT, 1994; McGRATH et al., 2007). Estudos sugerem que HPA provenientes da dieta podem estabelecer o processo de carcinogênese em diversos órgãos, como pulmão, trato gastrointestinal e mama (COYLE, 2004; ZHANG et al., 2009; XIA et al., 2010). Contudo, detectar o risco de câncer induzido por HPA proveniente exclusivamente da dieta é uma tarefa difícil, uma vez que não se encontram marcadores específicos que possam determinar a origem do contaminante. Assim, os HPA de origem alimentar se relacionam, sem distinção, com os HPA derivados de contaminação ambiental. Desta forma, destacamos a Tabela 2 como um compilado dos dados apresentados neste tópico, reunindo alternativas para reduzir ou prevenir a contaminação dos alimentos por HPA.

\section{1 Óleos vegetais}

Alimentos ricos em lipídios apresentam-se como fontes promissoras de contaminação por HPA, uma vez que estes contaminantes possuem natureza altamente lipofílica, facilitando assim a sua solubilidade nestes produtos alimentícios (DENNIS et al., 1991). Além disso, os lipídios atuam como veículo para os HPA, aumentando a velocidade de absorção intestinal destes agentes e, desta forma, aumentando o potencial de contaminação (STAVRIC; KLASSEN, 1994).

Os óleos vegetais comestíveis mais utilizados (soja, milho, girassol e oliva) podem apresentar elevado teor de HPA, que se originam por diversos fatores, como o crescimento de culturas em solo contaminado, a deposição de HPA durante o período de crescimento das culturas e a ineficiência dos processos de refino (TEIXEIRA et al., 2007; CAMARGO et al., 2011; FARHADIAN et al., 2012).

Utilizando óleos vegetais de milho, girassol e oliva, Dost e Ideli (2012) avaliaram a contaminação de nove tipos relevantes de HPA [fluoreno, fenantreno, antraceno, fluorantreno, pireno, benzo(b)fluoreno, benzo(a)anthracene, benzo(k)fluoranteno e benzo(a)pireno]. Todas as amostras apresentaram pelo menos a contaminação por três destes HPA, sendo os níveis de contaminação do óleo de milho alarmantes para o benzo(a)pireno, cuja concentração foi 10 vezes maior do que o valor de referência $(2,0 \mu \mathrm{g} / \mathrm{kg})$ para óleos e gorduras (EFSA, 2008); entretanto, nas amostras de óleo de oliva e de girassol, o benzo(a)pireno não foi detectado. O processo de secagem ao qual grãos de milho são submetidos é tido como a principal explicação para esses altos níveis de contaminação, pois se trata de secagem por meio de ar quente vindo diretamente da queima de lenha (LIMA et al., 2017). Apesar de o benzo(a) pireno, de maneira isolada, não ser mais considerado um marcador para HPA, ele apresenta propriedades carcinogênicas e mutagênicas (IARC, 2013); portanto, estes resultados sugerem que o óleo de milho pode representar um potencial risco à saúde dos consumidores em relação a este composto.

Tabela 2. Alternativas para reduzir ou prevenir a contaminação dos alimentos por HPA.

\begin{tabular}{|cl}
\hline \multicolumn{1}{c}{ Subtópicos } & \multicolumn{1}{c}{ Alternativas } \\
Óleos vegetais & Uso de carvão ativado no processo de refino \\
& Preferir a secagem artificial dos grãos \\
Laticínios & Evitar que vacas em lactação consumam pastagem contaminada \\
& Preferir leites com menor teor de gordura \\
Alimentos com alto teor de carboidratos & Aperfeiçoamento das técnicas de defumação de queijos \\
& Lavar a cana de açúcar antes da trituração \\
& Métodos de cocção que não exponham diretamente o alimento à chama \\
Modo de cocção & Expor a área com menos gordura para grelhar \\
& Marinar a carne com especiarias e ingredientes ácidos (evitar adição de óleo \\
& de cozinha) \\
& Preferir carvão de casca de coco ao carvão comum \\
Contaminação ambiental & Cultivar frutas e vegetais distantes de estradas e áreas industriais \\
& Preservação dos ambientes marinhos, principalmente em áreas de pesca \\
\hline
\end{tabular}


Uma grande preocupação se dá em torno do elevado nível de contaminação prévia por HPA nos grãos de soja produzidos em território nacional. O uso de secagem direta do material com ar aquecido é uma prática comum, que permite o contato direto entre os HPA presentes na fumaça e as sementes de soja; observe-se que estes compostos permanecem concentrados na superfície dos grãos e são transferidos para o óleo bruto (CAMARGO et al., 2012). Outro parâmetro importante é a procedência dos grãos, uma vez que o Brasil apresenta um grande território, com regiões e condições climáticas diferentes, em que a secagem artificial é sempre necessária (PUPIN; TOLEDO; 1996; MORET; CONTE, 2000; CAMARGO et al., 2011, 2012).

Entretanto, o processo de refino pode apresentar-se como um agente efetivo no decréscimo dos níveis de HPA, uma vez que, na etapa de branqueamento, o uso de carvão ativado promove a adsorção de parte destes compostos (LARSSON et al., 1987; CAMARGO; TOLEDO, 1998; TEIXEIRA et al., 2007). No Brasil, preferencialmente, é utilizado, como agente adsorvente, a terra de branqueamento, uma argila ativada que apresenta reduzido potencial de remoção dos HPA quando comparada ao carvão ativado (CAMARGO et al., 2012).

Não há legislação especifica, em nível nacional, sobre os níveis de HPA nos principais óleos comestíveis. Há apenas um nível máximo estabelecido pela Agência Nacional de Vigilância Sanitária (ANVISA) para benzo(a) pireno em óleo do bagaço de azeitona $(2,0 \mu \mathrm{g} / \mathrm{kg})$ e aromatizantes para defumação artificial $(0,03 \mu \mathrm{g} / \mathrm{kg})$, e uma Portaria do Ministério da Saúde para água potável (0,7 $\mu \mathrm{g} / \mathrm{L})$ (BRASIL, 2003, 2007, 2011).

Como parâmetro, temos a regulamentação europeia, que discorre sobre os níveis máximos de HPA em óleos e gorduras destinados ao consumo humano direto ou como ingrediente de alimentos, que estabelece 2,0 $\mathrm{mg} / \mathrm{kg}$ para o benzo(a)pireno e 10,0 $\mathrm{\mu g} / \mathrm{kg}$ para a soma de benzo(a) antraceno, benzo(a)pireno, benzo(b)fluoranteno e criseno, chamados de 4HPA. A legislação atual propõe que esses quatro compostos, em conjunto, passem a ser usados como possíveis marcadores da presença de HPA em alimentos e não apenas o benzo(a)pireno, como era de praxe (EFSA, 2008; EU, 2006; EC, 2011).

\subsection{Laticínios}

Os produtos lácteos estão presentes na dieta de diferentes países e culturas, sendo consumidos de várias formas e derivações. A apresentação desses produtos varia, principalmente, de acordo com o método de produção e o teor de gordura presente (FIDLER et al., 2001). Como o leite é um produto secretado pela glândula mamária, este pode exibir níveis significativos de vários xenobióticos, como, por exemplo, os HPA (SCHAUM et al., 2003). Desta forma, o leite pode ser visto como um indicador indireto para poluição ambiental, tendo como referência seu local de obtenção
(NACCARI et al., 2006). Certas propriedades de alguns HPA, como menor massa molecular e maior volatilidade, facilitam seu transporte e depósito sobre as pastagens, as quais, posteriormente, serão consumidas por vacas em lactação (GROVA et al., 2002; RYCHEN et al., 2005, 2008). Os HPA ingeridos podem ser transferidos para o leite, devido à sua capacidade de atravessar a barreira entre o sangue e a glândula mamária (GROVA et al., 2002).

No que diz respeito às técnicas de processamento e aquecimento (pasteurização, esterilização e ultra-tratamento por calor - UHT), estas corroboram diretamente com a elevação dos níveis de contaminação, uma vez que os HPA, de alto caráter lipofílico, produzidos por estas técnicas, são adicionalmente incorporados aos triglicerídeos, os principais componentes da gordura do leite (NACCARI et al., 2006, 2008).

A análise de amostras de leite submetidas a diferentes processamentos e tratamentos térmicos revelou diferenças entre os níveis de HPA (NACCARI et al., 2011). As amostras integrais submetidas ao tratamento UHT apresentaram maior índice total de contaminação por HPA, do que as pasteurizadas e in natura. Este fato pode estar diretamente relacionado à temperatura aplicada em cada processo, uma vez que, no processo de pasteurização, a amostra é submetida à temperatura de $72^{\circ} \mathrm{C}$ a $75^{\circ} \mathrm{C}$, de 15 a 20 segundos, enquanto que, no processo UHT, o leite é submetido a temperaturas entre $130^{\circ} \mathrm{C}$ e $150^{\circ} \mathrm{C}$, durante 2 a 4 segundos (RODRIGUES et al., 2013). Desta forma, sugere-se que a exposição a altas temperaturas contribua para níveis de contaminação mais elevados.

Além disso, certos HPA só foram encontrados em amostras de leite pasteurizado e UHT, o que sugere que o processamento e o tratamento térmico favorecem a formação de novos compostos (NACCARI et al., 2011; ABDEL-SHAFY; MANSOUR, 2016). O fato do leite em seu estado natural apresentar níveis de contaminação menores do que as amostras de leite pasteurizado e UHT indica que esse resultado é soma dos HPA provenientes da contaminação ambiental e os produzidos pelo processamento e tratamento térmico (SIMONEIT, 2002). Visto que o leite in natura obviamente não foi submetido ao processo de aquecimento, sua contaminação foi atribuída somente à transferência de HPA do solo para os animais em lactação e à contaminação atmosférica (GROVA et al., 2002; RYCHEN et al., 2005).

Quando se leva em consideração a concentração de gorduras de amostras de leite submetidas ao tratamento UHT, as amostras com menor teor de gordura (semidesnatado) apresentaram menores índices de contaminação, confirmando que os níveis de HPA relacionam-se intimamente com o teor de gordura do alimento (DOREMIRE et al., 1979; D'MELLO, 2003; NACCARI et al., 2011).

A realização de estudos que relacionem o conteúdo de HPA em derivados lácteos é de grande importância. Comparações do perfil de HPA encontrados em amostras 
de queijos defumados artesanalmente detectaram variação nos resultados quanto à posição dos queijos no defumador, o que permite dizer que um aperfeiçoamento das técnicas de defumação pode contribuir para a redução do grau de contaminação por HPA, pois as amostras colocadas na direção da fumaça apresentaram maiores níveis de contaminação (GUILLÉN et al., 2011). Vale ressaltar que a natureza da matéria vegetal usada para defumação parece exercer influência sobre o tipo de HPA formado, especialmente em derivados alquil e alguns HPA leves (GUILLÉN et al., 2007).

\subsection{Alimentos com alto teor de carboidratos}

Dentre os trabalhos que correlacionam alimentos ricos em açúcares, presença de HPA e produtos de origem nacional, podemos citar a análise destes contaminantes no caldo de cana e na rapadura.

Foram coletadas amostras de caldo de cana nos municípios paulistas de Ribeirão Preto e Campinas, buscando determinar a concentração de benzo(a)antraceno, benzo(b) fluorantreno, benzo(k)fluorantreno e benzo(a)pireno, além da influência do processo de queima da cana no nível destes constituintes (TFOUNI et al., 2009). As amostras coletadas na época em que a cana é queimada mostraram resultados quatro vezes maiores do que as coletadas na entressafra, apontando a queima da cana com uma importante fonte de contaminação. Outro aspecto que deve ser mencionado é a possível remoção mecânica de parte da contaminação, já que, antes de triturar a cana de açúcar, o produto é lavado. Tal processo pode contribuir para a retirada de parte dos HPA de origem ambiental, uma vez que a simples pressão exercida pela água pode remover uma camada superficial de fuligem, diminuindo, assim, a quantidade bruta de contaminantes (SILVA et al., 2008; TFOUNI et al., 2009).

A rapadura, produzida a partir do caldo de cana-de-açúcar, é um alimento muito presente na mesa brasileira e está incluída no cardápio de escolas públicas, por fazer parte do PNAE (Programa Nacional de Alimentação Escolar) (WEIS et al., 2004). Compostos mutagênicos e carcinogênicos, incluindo os HPA, podem contaminar este alimento durante o processo de produção (ZAMPERLINI et al., 2000; CAMARGO; TOLEDO, 2002). Tendo essa possibilidade em vista, Silva et al. (2011) avaliaram a presença de 16 HPA em amostras obtidas de diferentes Regiões do país (Nordeste, Sudeste e Centro-Oeste).

Em 95\% das amostras, foram encontrados HPA, sendo o pireno o composto mais comum, seguido pelo antraceno. As maiores concentrações de HPA foram encontradas na Região Sudeste, seguida da Região Centro-Oeste e da Região Nordeste, com menor índice. A significativa diferença encontrada entre os níveis de HPA nas diferentes regiões do país coloca mais uma vez o processo de queima da cana como uma variável importante, pois essa é uma prática cuja frequência varia de acordo com a região e que pode influenciar os níveis de ingestão de HPA. O controle de qualidade, antes mesmo de o produto ser processado, é muito importante para identificar e controlar as fontes de HPA; contudo, a larga variação encontrada entre as concentrações de amostras da mesma região indica que essa é uma prática ausente (SILVA et al., 2011).

Quanto aos aspectos legais, o Brasil não dispõe de uma legislação específica sobre os níveis toleráveis de HPA em produtos ricos em carboidratos; por isso, as amostras de caldo de cana analisadas por Tfouni et al. (2009) foram comparadas com os limites permitidos para benzo(a)pireno em água potável (BRASIL, 2011) e, com base nessa comparação, as amostras foram consideradas seguras para o consumo. Entretanto, a análise ficou limitada a um composto em particular, não havendo um comparativo para os demais HPA encontrados. Por se assemelharem quanto ao alto teor de carboidratos e, na ausência de parâmetros específicos, a legislação europeia (EU, 2006), que diz respeito à concentração de HPA presentes em alimentos processados à base de cereais, foi utilizada por Silva e colaboradores (2011), para análise da rapadura. Tal parâmetro, contudo, além de não ser específico, utiliza a legislação de países que não incluem a rapadura como um produto de consumo interno expressivo.

Os níveis de HPA em cereais são baixos quando comparados aos níveis de HPA em óleos e gorduras, por exemplo; contudo, sua grande presença na dieta de vários povos faz dessa classe de alimentos um contribuinte significativo para o nível de ingestão alimentar desses contaminantes. Na China, foi constatado que a exposição diária total da população a HPA, através da ingestão de arroz, foi superior ou semelhante à exposição através do consumo de outros cereais, e considerada equivalente à dieta total, em outros países (DING et al., 2012). Apesar da poluição ambiental na China ser maior, a situação no Brasil merece atenção, visto que o arroz é o principal produto da base nutricional da população brasileira, com um consumo interno estimado em quase 12 milhões de toneladas para o período 2015/2016 (CONAB, 2015).

Uma estimativa relacionando o consumo de HPA presentes no arroz à faixa etária apontou as crianças como o grupo mais sensível à exposição de HPA (DING et al., 2012). Desta forma, torna-se de grande importância o desenvolvimento de estudos relacionando o consumo de HPA a outros alimentos, como o chocolate, que é um ingrediente base de inúmeras preparações e amplamente consumido por todas as faixas etárias, sobretudo pelas crianças. Tal estudo se justifica, principalmente, se levamos em consideração que seu método de obtenção 
pode gerar HPA, por requer processos complexos, como técnicas de secagem e torra.

Na avalição realizada pelo European Food Safety Authority (EFSA, 2008), de todos os alimentos analisados, o chocolate e a manteiga de cacau estavam entre os que apresentaram níveis mais significativos para benzo(a) pireno, junto com a carne grelhada, o chá desidratado e os moluscos bivalves, como ostras e mexilhões.

Os resultados existentes tornam-se preocupantes no que diz respeito aos altos índices de contaminação (ZIEGENHALS et al., 2009; KUMARI et al., 2012), que podem representar um risco para a saúde dos consumidores. Dessa forma, se faz necessário o desenvolvimento de estudos adicionais, além da avaliação de medidas eficazes para prevenir contaminações de maior magnitude em chocolate e derivados, bem como regulamentar um limite para o percentual dos principais HPA nestes produtos.

\subsection{Modo de cocção}

O modo de cocção ao qual o alimento é submetido relaciona-se diretamente com os índices de ingestão de HPA. Diferenças são encontradas tanto devido ao modo de preparo quanto às variações dentro do método escolhido (Figura 1).

Dentre os métodos de cocção mais relacionados à formação de HPA estão fritar, grelhar e assar (WHO, 2005; FERGUSON, 2010; ROSE et al., 2015). No que diz respeito a grelhar ou assar, a literatura descreve que os alimentos assados, de modo geral, apresentam menor formação de HPA que os grelhados. A concentração de HPA nos assados a gás é ainda menor do que nos alimentos assados utilizando carvão vegetal. (CHUNG et al., 2011).
A justificativa para isso pode vir da pirólise da gordura derretida que goteja sobre o carvão durante a cocção, levando à subsequente deposição de HPA através da fumaça (LIJINSKY; ROSS, 1967; DOST; IDELI, 2012).

A comparação do carvão obtido por madeira com o obtido por casca de coco demostrou índices maiores de HPA para alimentos grelhados com a utilização do carvão da madeira (VIEGAS et al., 2012). A possível explicação para o fato é que o carvão de casca de coco promove a menor liberação de chama e fumaça, além da possibilidade de absorver a gordura que goteja durante a cocção. Desse modo, o tipo de carvão escolhido pode ser um fator que colabora diretamente na redução dos níveis destes contaminantes.

Quanto ao processo de fritura, um percentual adicional de HPA deve ser considerado, uma vez que o óleo utilizado pode apresentar contaminação prévia (TEIXEIRA et al., 2007; CAMARGO et al., 2011, 2012) e este ainda será submetido a altas temperaturas, processo que poderá aumentar consideravelmente os níveis destes contaminantes (PURCARO et al., 2006).

A escolha da fonte térmica empregada (calor direto ou indireto) também está associada à formação de HPA durante o processo de cocção. Amostras de pão torrado submetidas a métodos diretos, em que é utilizada a chama como fonte de calor para tostagem, obtiveram índices de contaminação mais significativos que os indiretos, nos quais a geração de calor se faz na ausência de chama, ocorrendo, por exemplo, a partir de uma resistência elétrica. Este fato pode ser explicado pelo fluxograma apresentado na Figura 2, uma vez que, na queima de forma direta, a contaminação por HPA é gerada por pirólise de macronutrientes e, principalmente,

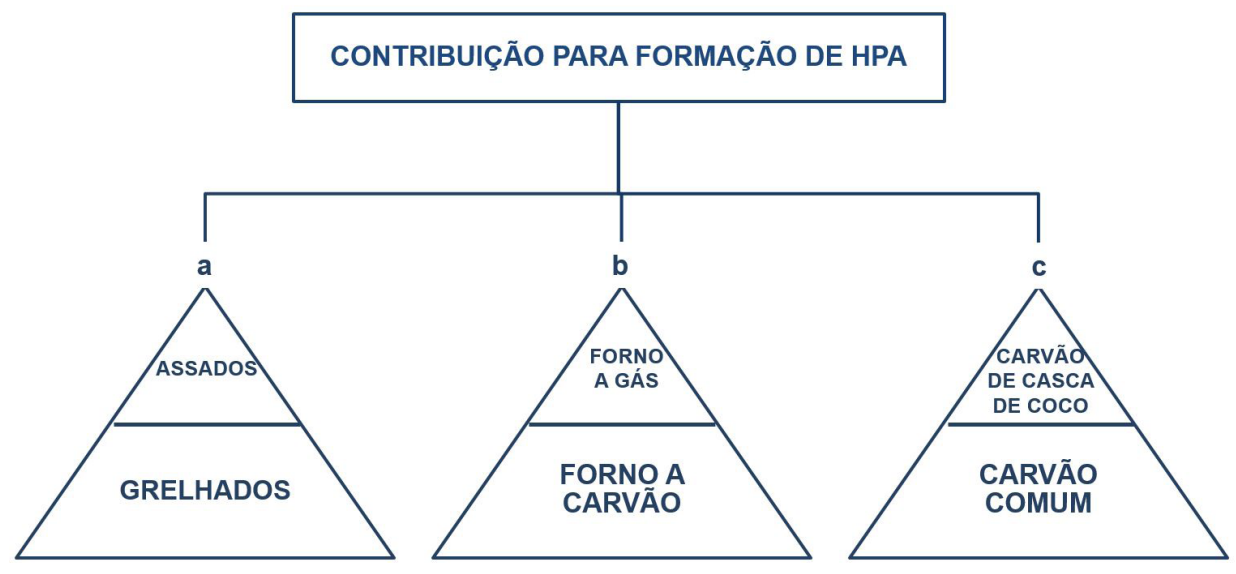

a. Alimentos assados apresentam menor formação de HPA em comparação aos grelhados.

b. A fumaça, gerada pela pirólise da gordura que derrete e goteja sobre o carvão durante a cocção, deposita HPA sobre o alimento.

c. O carvão de casca de coco promove menor liberação de chama e fumaça, além de absorver a gordura que goteje sobre ele.

Figura 1. Níveis de contribuição para formação de HPA de acordo com o método de cocção. 


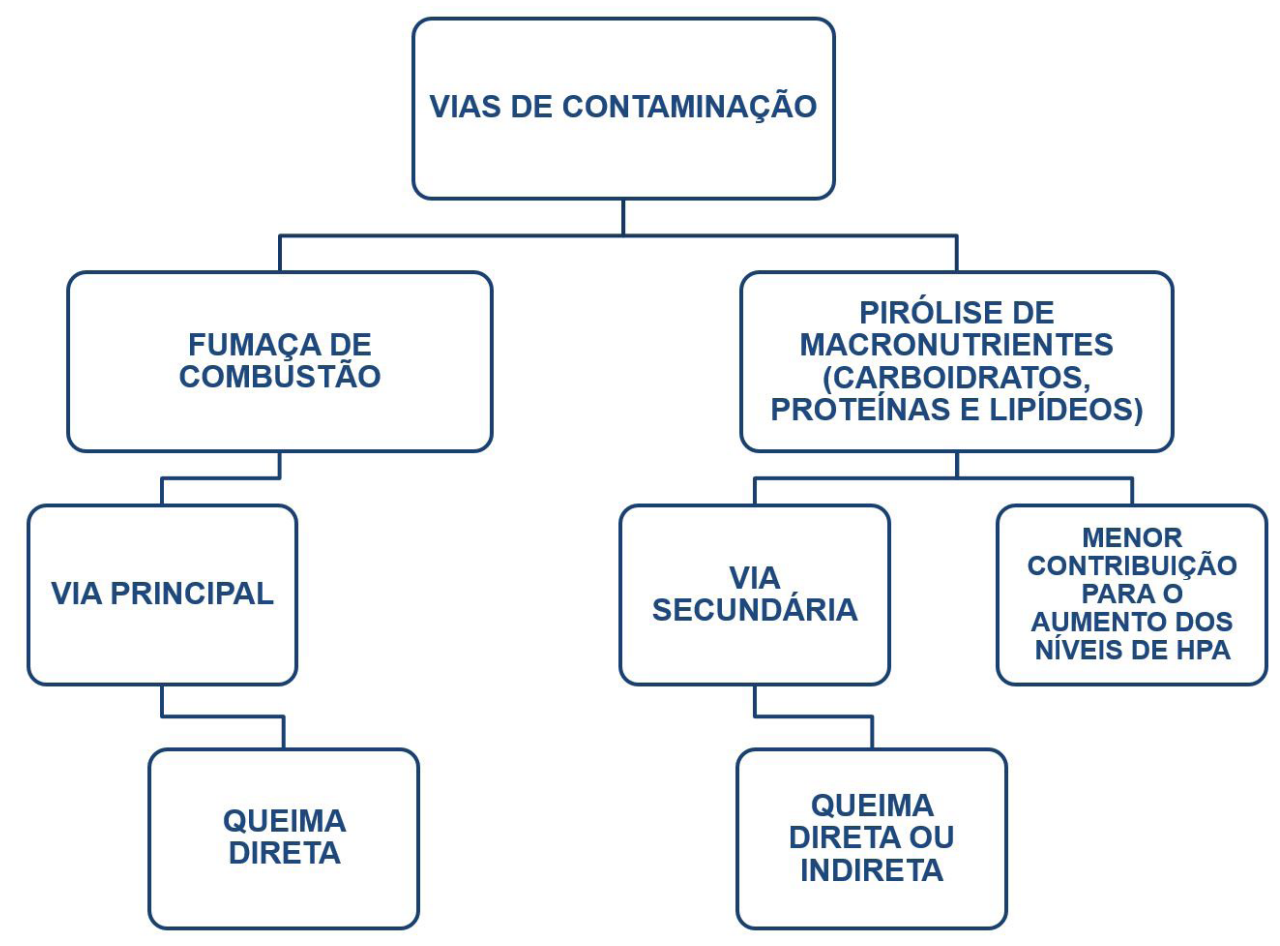

Figura 2. Vias de contaminação geradas por calor direto ou indireto.

por deposição de HPA a partir da fumaça de combustão, enquanto os métodos indiretos se restringem ao processo de pirólise, menos associados à formação de fumaça (REY-SALGUEIRO et al., 2008).

O tratamento a que um alimento é submetido antes da cocção também pode influenciar, positiva ou negativamente, no teor de HPA presentes no produto final, pois certos condimentos funcionam como inibidores da formação de alguns compostos carcinogênicos (BRITT et al., 2004; SHON et al., 2004; NURSTEN, 2005; GIBIS, 2007; KEHLET et al., 2011). Estudos indicam que marinar a carne antes de grelhá-la reduz a formação de HPA (FARHADIAN et al., 2012). Essa redução pode ser devida à presença de especiarias conhecidas pela sua atividade antioxidante, especialmente o alho e a cebola, que têm sido relatados como inibidores da formação de alguns compostos carcinogênicos (NAKAYAMA et al., 1993; SHON et al., 2004; SMITH et al., 2008; ALOMIRAH et al., 2011; JANOSZKA, 2011). Em contrapartida, a concentração de HPA em amostras de carne marinada contendo óleo de cozinha (como um ingrediente) é mais elevada, devido à sua absorção (FARHADIAN et al., 2012).

No entanto, a adição de ingrediente ácido, como suco de limão ou de tamarindo, nos tratamentos contendo óleo de cozinha reduz a formação de HPA. Seu efeito protetor poderia ser evidenciado se aplicado isoladamente, uma vez que a presença de óleo no tratamento diminuiu o efeito redutor do agente ácido quanto à formação de HPA, o que pode ser devido a uma fragilização das condições ácidas (BRIEN et al., 1998; NURSTEN, 2005; GIBIS, 2007).

\subsection{Contaminação ambiental}

Um ponto muito importante que deve ser considerado, quando se avalia a presença de HPA em alimentos, é a sua possível exposição a contaminantes de origem ambiental, uma vez que a poluição é um dos principais meios de contaminação de diversos gêneros alimentícios (DONG; LEE, 2009; AQUILINA et al., 2010; ZHANG et al., 2010).

Em frutas e hortaliças, por exemplo, a presença desses compostos vem principalmente da deposição de partículas de poluição do ar em suas superfícies, enquanto peixes e invertebrados marinhos podem conter naturalmente pequenas quantidades de diferentes HPA absorvidos do ambiente no qual estão inseridos (LARSSON, 1986). A fonte desse tipo de contaminação geralmente provém da poluição atmosférica (resultante da queima incompleta de combustíveis), dos vazamentos de óleos e dos detritos de origem doméstica e industrial, dispensados em efluentes que atingirão o oceano (DONG; LEE, 2009; KATAYAMA et al., 2010; AZIZ et al., 2014).

Quando avaliamos a exposição de vegetais podemos dizer que os níveis de contaminação dependem do local de cultivo, pois regiões mais poluídas tendem a ofertar alimentos mais contaminados. Amostras de localidades altamente industrializadas ou cultivadas perto de estradas, em geral, apresentam níveis mais elevados que as amostras provenientes das áreas rurais (LARSSON, 1986; 
CAMARGO; TOLEDO, 2003). As características do alimento correspondem a outro fator influente sobre os níveis de contaminação. Alimentos com uma grande área de superfície exposta estão mais propensos à contaminação, uma vez que os HPA tendem a acumular-se mais nas áreas externas dos vegetais, como na casca das frutas e nas folhas (LARSSON, 1986).

Estudos visando estimar a exposição alimentar dos brasileiros à contaminação por HPA determinaram expressivo potencial carcinogênico em amostras de maçã, pera, uva, alface, tomate e couve, bem como a influência da poluição ambiental sobre os níveis de HPA nestes produtos (LARSSON, 1986). As hortaliças apresentaram níveis maiores de HPA que os valores encontrados nas frutas. A alface revelou os níveis mais elevados, entre todas as amostras, seguida pelo tomate, couve, maçã, uva e pera. Estes resultados já eram esperados devido à grande área de superfície da alface, quando comparada aos outros produtos (LARSSON, 1986; CAMARGO; TOLEDO, 2003).

Quanto aos produtos derivados da pesca, os resultados indicam a presença de HPA em todas as amostras frescas, entre as quais: peixes de água doce, peixes de água salgada, moluscos, algas, camarões e caranguejos (ZHANG et al., 2010). No entanto, todos os produtos avaliados apresentaram-se seguros para consumo, pois os níveis encontrados não ultrapassaram os limites fixados pela União Europeia (EU, 2005). Contudo, certos produtos de pesca, como ostras e mexilhões, por não apresentarem a capacidade de biotransformar os HPA podem acumulá-los em seus organismos (MEADOR et al., 1995).

Levando em consideração, ainda, a imensa faixa de compostos aromáticos polinucleares que podem ser encontrados em alimentos, a exposição e os riscos à saúde podem ser significativamente maiores. Os HPA presentes na atmosfera encontram-se entre a fase gasosa e o material particulado. Dessa forma, o transporte de HPA pelo ar é influenciado pela sua volatilidade, uma vez que os compostos de dois a três anéis aromáticos, geralmente, estão na fase de vapor; os de quatro anéis apresentam-se numa fase intermediária e os de cinco ou mais anéis são encontrados predominantemente particulados (CONDE et al., 2004). Tais propriedades físico-químicas permitem que os HPA sejam transportados por longas distâncias antes de serem adsorvidos em material particulado (GODOI et al., 2004; LIN et al., 2005). Além disso, a baixa reatividade química e a hidrofobicidade facilitam ainda mais uma distribuição global dos HPA, pois permitem que estes se acumulem tanto em ambientes aquáticos como terrestres (GODOI et al., 2004).

Todos estes fatores podem contribuir para a persistência e a acumulação de HPA na cadeia alimentar. Assim, cidades com uma intensa emissão de poluentes devem ficar especialmente mais atentas aos riscos causados pela exposição a HPA, independentemente da origem desses contaminantes, visto que a contaminação atmosférica, além de ser absorvida pelas vias respiratórias e pela pele, também pode depositar-se nos alimentos (XIA et al., 2010).

\section{Conclusão}

A natureza do alimento é um fator primordial para o nível de contaminação por HPA, sendo o percentual lipídico o mais relevante. Dentro os grupos apresentados, destacam-se os óleos vegetais, e considera-se importante o percentual de gordura presente nos laticínios e em produtos cárneos. O modo de preparo também pode influenciar significativamente os níveis de HPA presentes nos alimentos, podendo apresentar as condições para formação desses compostos e sua consequente ingestão. Especialmente, nos alimentos grelhados, vastamente consumidos, encontra-se grande contaminação, o que faz destes alimentos um risco em potencial. Quanto ao tratamento empregado antes da cocção, estudos revelam que ambiente ácido e certos condimentos antioxidantes exercem o papel de inibidores da formação desses compostos, contrabalanceando os efeitos negativos causados pelo modo de preparo.

É crescente o número de trabalhos na área de identificação e doseamento de HPA em alimentos; contudo, a legislação brasileira é pouco abrangente, visto que cobre apenas alguns produtos e enfatiza o benzo(a)pireno, o que direciona os estudos desenvolvidos nacionalmente a se adaptarem a regulamentações de outros países. Estas, muitas vezes, não se encaixam nos hábitos alimentares da nossa população, o que pode estar prejudicando a estimativa de níveis de exposição mais realistas. Tal necessidade de ampliação das normas para produtos nacionais se torna ainda mais evidente quando este tema é visto como uma questão de Segurança de Alimentos.

\section{Referências}

ABDEL-SHAFY, H. I.; MANSOUR, M. S. M. A review on polycyclic aromatic hydrocarbons: source, environmental impact, effect on human health and remediation. Egyptian Journal of Petroleum, v. 25 , n. 1, p. 107-123, 2016. http://dx.doi.org/10.1016/j. ejpe.2015.03.011

ALOMIRAH, H.; AL-ZENKI, S.; AL-HOOTI, S.; ZAGHLOUL, S.; SAWAYA, W.; AHMED, N.; KANNAN, K. Concentrations and dietary exposure to polycyclic aromatic hydrocarbons (PAHs) from grilled and smoked foods. Food Control, v. 22, n. 12, p. 2028-2035, 2011. http://dx.doi.org/10.1016/j.foodcont.2011.05.024.

AQUILINA, N. J.; DELGADO-SABORIT, J. M.; MEDDINGS, C.; BAKER, S.; HARRISON, R. M.; JACOB, P.; WILSON, M.; YU, L.; DUAN, M.; BENOWITZ, N. L. Environmental and biological monitoring of exposures to PAHs and ETS in the general population.

Environment International, v. 36, n. 7, p. 763-771, 2010. PMid:20591483. http://dx.doi.org/10.1016/j.envint.2010.05.015. 
Presença de hidrocarbonetos policíclicos aromáticos em produtos alimentícios e a sua relação com o método de cocção e a natureza do alimento Paz, A. P. S. et al.

AZIZ, F.; SYED, J. H.; MALIK, R. N.; KATSOYIANNIS, A.; MAHMOOD, A.; LI, J.; ZHANG, G.; JONES, K. C. Occurrence of polycyclic aromatic hydrocarbons in the Soan River, Pakistan: insights into distribution, composition, sources and ecological risk assessment. Ecotoxicology and Environmental Safety, v. 109, p. 77-84, 2014. PMid:25241944. http://dx.doi.org/10.1016/j. ecoenv.2014.07.022.

BALBANI, A. P. S.; BUTUGA, O. Contaminação biológica de alimentos. Pediatria, v. 23, p. 320-328, 2001.

BETTIN, S. M.; FRANCO, D. W. Hidrocarbonetos policíclicos aromáticos (HPAs) em aguardentes. Food Science and Technology, v. 25, n. 2, p. 234-238, 2005. http://dx.doi.org/10.1590/S010120612005000200008.

BOBEDA, C. R. R.; GODOY, R. L. O.; PACHECO, S.; BORGUINI, R. G.; TORQUILHO, H. S. Avaliação da presença de hidrocarbonetos policíclicos aromáticos (HAPs) em amostras de mexilhões importados do Chile. Higiene Alimentar, v. 27, p. 218-219, 2013.

BRASIL. Resolução RDC n 281, de 6 de outubro de 2003. Exige como procedimento de importação para "aceite de orujo de oliva" ou óleo de bagaço e ou caroço de oliva, sem prejuízo da documentação exigida para este fim, a apresentação do laudo de análise do produto quanto à presença de hidrocarbonetos policíclicos aromáticos, especificamente o alfa-benzopireno, com identificação do lote e ou data de produção ou fabricação. Diário Oficial [da] República Federativa do Brasil, Brasília, DF, 8 out. 2003.

BRASIL. Resolução RDC n² 2, de 15 de janeiro de 2007. Diário Oficial [da] República Federativa do Brasil, Brasília, DF, 17 jan. 2007

BRASIL. Portaria MS n² 2914, de 12 de dezembro de 2011. Dispõe sobre os procedimentos de controle e de vigilância da qualidade da água para consumo humano e seu padrão de potabilidade. Diário Oficial [da] República Federativa do Brasil, Brasília, DF, 12 dez. 2011. Seção 1, p. 266-270.

BRIEN, J. O.; NURSTEN, H. E.; CRABBL, M. J. C.; AMES, J. M. The Maillard reaction in food and medicine. Reading: University of Reading, The Royal Society of Chemistry, 1998.

BRITT, P. F.; BUCHANAN, A. C.; OWENS, C. V. Jr; TODD SKEEN, $J$. Does glucose enhance the formation of nitrogen containing polycyclic aromatic compounds and polycyclic aromatic hydrocarbons in the pyrolysis of proline? Fuel, v. 83, n. 11-12, p. 1417-1432, 2004. http://dx.doi.org/10.1016/j.fuel.2004.02.009.

CAMARGO, M. C. R.; ANTONIOLLI, P. R.; VICENTE, E.; TFOUNI, S. A. V. Polycyclic aromatic hydrocarbons in Brazilian commercial soybean oils and dietary exposure. Food Additives \& Contaminants B, v. 4, n. 2, p. 152-159, 2011. PMid:24785726. http://dx.doi.org/10.1080/19393210.2011.585244.

CAMARGO, M. C. R.; ANTONIOLLI, P. R.; VICENTE, E. Evaluation of polycyclic aromatic hydrocarbons content in different stages of soybean oils processing. Food Chemistry, v. 135, n. 3, p.
937-942, 2012. PMid:22953808. http://dx.doi.org/10.1016/j. foodchem.2012.06.031.

CAMARGO, M. C. R.; TOLEDO, M. C. F. Polycyclic aromatic hydrocarbon contamination in different commodity groups. Brazilian Journal of Food Technology, v. 5, p. 19-26, 2002.

CAMARGO, M. C. R.; TOLEDO, M. C. F. Polycyclic aromatic hydrocarbons in Brazilian vegetables and fruits. Food Control, v. 14, n. 1, p. 49-53, 2003. http://dx.doi.org/10.1016/S09567135(02)00052-X

CAMARGO, M. S. F. O.; TOLEDO, M. C. F. Efeito do processamento na contaminação de óleo refinado de milho por benzo(a)pireno. Brazilian Journal of Food Technology, v. 1, p. 97-106, 1998.

CARUSO, M. S. F.; ALABURDA, J. Hidrocarbonetos policíclicos aromáticos - benzo(a)pireno: uma revisão. Revista do Instituto Adolfo Lutz, v. 67, p. 1-27, 2008.

CHUNG, S. Y.; YETTELLA, R. R.; KIM, J. S.; KWON, K.; KIM, M. C.; MIN, D. B. Effects of grilling and roasting on the levels of polycyclic aromatic hydrocarbons in beef and pork. Food Chemistry, v. 129, n. 4, p. 1420-1426, 2011. http://dx.doi. org/10.1016/j.foodchem.2011.05.092.

COMPANHIA NACIONAL DE ABASTECIMENTO - CONAB. Perspectivas para a agropecuária. Brasília: CONAB, 2015. v. 3 : Safra 2015/2016 (Produtos de Verão). Disponível em: <http://www. conab.gov.br/OlalaCMS/uploads/arquivos/15_09_24_11_44_50_ perspectivas_agropecuaria_2015-16_-_produtos_verao.pdf > Acesso em: 1 dez. 2016.

CONDE, F. J.; AYALA, J. H.; AFONSO, A. M.; GONZÁLEZ, V. Optimization of a sampling method to determine polycyclic aromatic hydrocarbons in smoke from incomplete biomass combustion. Analytica Chimica Acta, v. 524, n. 1-2, p. 287-294, 2004. http://dx.doi.org/10.1016/j.aca.2004.04.063.

COYLE, Y. M. The effect of environment on breast cancer risk. Breast Cancer Research and Treatment, v. 84, n. 3, p. 273-288, 2004. PMid:15026625. http://dx.doi.org/10.1023/ B:BREA.0000019964.33963.09.

DANYI, S.; BROSE, F.; BRASSEUR, C.; SCHNEIDER, Y.-J.; LARONDELLE, Y.; PUSSEMIER, L.; ROBBENS, J.; DE SAEGER, S.; MAGHUIN-ROGISTER, G.; SCIPPO, M.-L. Analysis of EU priority polycyclic aromatic hydrocarbons in food supplements using high performance liquid chromatography coupled to an ultraviolet, diode array or fluorescence detector. Analytica Chimica Acta, v. 633, n. 2, p. 293-299, 2009. PMid:19166736. http://dx.doi.org/10.1016/j.aca.2008.11.049.

DENNIS, M. J.; MASSEY, R. C.; CRIPPS, G.; VENN, I.; HOWARTH, N.; LEE, G. Factors affecting the polycyclic aromatic hydrocarbon content of cereals, fats and other food products. Food Additives and Contaminants, v. 8, n. 4, p. 517-530, 1991. PMid:1806403. http://dx.doi.org/10.1080/02652039109374004.

DING, C.; NI, H.-G.; ZENG, H. Parent and halogenated polycyclic aromatic hydrocarbons in rice and implications for human health 
Presença de hidrocarbonetos policíclicos aromáticos em produtos alimentícios e a sua relação com o método de cocção e a natureza do alimento Paz, A. P. S. et al.

in China. Environmental Pollution, v. 168, p. 80-86, 2012. PMid:22595763. http://dx.doi.org/10.1016/j.envpol.2012.04.025.

D'MELLO, J. P. F. Food safety: contaminants and toxins. Wallingford: CABI Publishing, 2003.

DONG, T. T.; LEE, B. K. Characteristics, toxicity, and source apportionment of polycylic aromatic hydrocarbons (PAHs) in road dust of Ulsan, Korea. Chemosphere, v. 74, n. 9, p. 1245-1253, 2009. PMid:19103459. http://dx.doi.org/10.1016/j. chemosphere.2008.11.035

DOREMIRE, M. E.; HARMON, G. E.; PRATT, D. E. 3.4-BenzoPyrene in charcoal grilled meats. Journal of Food Science, $v$. 44, n. 2, p. 622, 1979. http://dx.doi.org/10.1111/j.1365-2621.1979. tb03851.x.

DOST, K.; IDELI, C. Determination of polycyclic aromatic hydrocarbons in edible oils and barbecued food by HPLC/UVVis detection. Food Chemistry, v. 133, n. 1, p. 193-199, 2012. http://dx.doi.org/10.1016/j.foodchem.2012.01.001.

ENVIRONMENTAL PROTECTION AGENCY - US EPA. Locating and estimating air emissions from sources of polycyclic organic matter. North Carolina: EPA, 1998. EPA-454/R-98/014.

EUROPEAN COMMISSION - EC. Commission Regulation (EU) No. 835/2011 of 19 August 2011 amending Regulation EC No. $1881 / 2006$ as regards maximum levels for polycyclic aromatic hydrocarbons in foodstuffs. Official Journal of the European Union, Luxemburgo, 20 aug. 2011. v. L215, p. 4-8.

EUROPEAN FOOD SAFETY AUTHORITY - EFSA. Scientific opinion of the panel on contaminants in the food chain on a request from the European Commission on polycyclic aromatic hydrocarbons in food. EFSA, v. 724, p. 1-114, 2008.

EUROPEAN UNION - EU. Commission Regulation (EC) No 208/2005 of 4 February 2005. Amending Regulation No. 466/2001. Regards Polycyclic Aromatic Hydrocarbons. Official Journal of the European Union, Luxemburgo, 8 feb. 2005. v. 34, p. 3-5.

EUROPEAN UNION - EU. Commission Regulation No. 1881/2006 of 19 December 2006. Maximum levels for certain contaminants in foodstuffs. Official Journal of the European Union, Luxemburgo, 20 dec. 2006. v. 364, p. 5-24.

FALCÓ, G.; DOMINGO, J. L.; LLOBET, J. M.; TEIXIDÓ, A.; CASAS, C.; MÜLLER, L. Polycyclic aromatic hydrocarbons in foods: human exposure through the diet in Catalonia, Spain. Journal of Food Protection, v. 66, p. 2325-2331, 2003. PMid:14672232. http://dx.doi.org/10.4315/0362-028X-66.12.2325.

FAN, R.; DONG, Y.; ZHANG, W.; WANG, Y.; YU, Z.; SHENG, G.; $F U, J$. Fast simultaneous determination of urinary 1-hydroxypyrene and 3-hydroxybenzo[a]pyrene by liquid chromatography-tandem mass spectrometry. Journal of Chromatography B: Analytical Technologies in the Biomedical and Life Science, v. 836, n. 1-2, p. 92-97, 2006. PMid:16617038.
FARHADIAN, A.; JINAP, S.; ABAS, F.; SAKAR, Z. I. SAKAR ZI. Determination of polycyclic aromatic hydrocarbons in grilled meat. Food Control, v. 21, n. 5, p. 606-610, 2010. http://dx.doi. org/10.1016/j.foodcont.2009.09.002.

FARHADIAN, A.; JINAP, S.; FARIDAH, A.; ZAIDUL, I. S. M. ZAIDUL ISM. Effects of marinating on the formation of polycyclic aromatic hydrocarbons (benzo[a]pyrene, benzo[b]fluoranthene and fluoranthene) in grilled beef meat. Food Control, v. 28, n. 2, p. 420-425, 2012. http://dx.doi.org/10.1016/j.foodcont.2012.04.034.

FERGUSON, L. R. Meat and cancer. Meat Science, v. 84, n. 2, p. 308-313, 2010. PMid:20374790. http://dx.doi.org/10.1016/j. meatsci.2009.06.032.

FIDLER, N.; SAUERWALD, T. U.; DEMMELMAIR, H.; KOLETZKO, B. Fat content and fatty acid composition of fresh, pasteurized, or sterilized human milk. Advances in Experimental Medicine and Biology, v. 501, p. 485-495, 2001. PMid:11787720. http:// dx.doi.org/10.1007/978-1-4615-1371-1_60.

GIBIS, M. Effect of oil marinades with garlic, onion and lemon juice on the formation of heterocyclic aromatic amines in fried beef patties. Journal of Agricultural and Food Chemistry, v. 55, n. 25, p. 10240-10247, 2007. PMid:17988088. http://dx.doi. org/10.1021/jf071720t.

GILBERT, J. The fate of environmental contaminants in the food chain. The Science of the Total Environment, v. 143, n. 1, p. 103-111, 1994. PMid:8202695. http://dx.doi.org/10.1016/00489697(94)90536-3.

GODOI, A. F.; RAVINDRA, K.; GODOI, R. H.; ANDRADE, S. J.; SANTIAGO-SILVA, M.; VAN VAECK, L.; VAN GRIEKEN, R. Fast chromatographic determination of polycyclic aromatic hydrocarbons in aerosol samples from sugar cane burning. Journal of Chromatography A, v. 1027, n. 1-2, p. 49-53, 2004. PMid:14971483. http://dx.doi.org/10.1016/j.chroma.2003.10.048.

GROVA, N.; FEIDT, C.; LAURENT, C.; RYCHEN, G. $\left[{ }^{14} \mathrm{C}\right]$ Milk, urine and faeces excretion kinetics in lactating goats after an oral administration of $\left[{ }^{14} \mathrm{C}\right]$ polycyclic aromatic hydrocarbons. International Dairy Journal, v. 12, p. 1025, 2002. http://dx.doi. org/10.1016/S0958-6946(02)00126-7.

GUILLÉN, M. D.; PALENCIA, G.; IBARGOITIA, M. L.; FRESNO, M.; SOPELANA, P. Contamination of cheese by polycyclic aromatic hydrocarbons in traditional smoking. Influence of the position in the smokehouse on the contamination level of smoked cheese. Journal of Dairy Science, v. 94, n. 4, p. 1679-1690, 2011. PMid:21426955. http://dx.doi.org/10.3168/jds.2010-3647.

GUILLÉN, M. D.; PALENCIA, G.; SOPELANA, P.; IBARGOITIA, M. $L$. Occurrence of polycyclic aromatic hydrocarbons in artisanal palmero cheese smoked with two types of vegetable matter. Journal of Dairy Science, v. 90, n. 6, p. 2717-2725, 2007. PMid:17517711. http://dx.doi.org/10.3168/jds.2006-452.

HARVEY, R. G. Polycyclic aromatic hydrocarbons and cancer. American Scientist, v. 70, n. 4, p. 386-393, 1982. PMid:7149435. 
Presença de hidrocarbonetos policíclicos aromáticos em produtos alimentícios e a sua relação com o método de cocção e a natureza do alimento Paz, A. P. S. et al.

HOWARD, J. W.; FAZIO, T. Analytical methodology and reported findings of polycyclic aromatic hydrocarbons in foods. Journal Association of Official Analytical Chemists, v. 63, n. 5, p. 1077-1104, 1980. PMid:6997260.

INTERNATIONAL AGENCY FOR RESEARCH ON CANCER - IARC. Agents classified by the IARC monographs. Lyon: IARC, 2013. v. 1. Disponível em: <http://monographs.iarc.fr/ENG/Classification/ ClassificationsAlphaOrder.pdf>. Acesso em: 1 dez. 2016.

INTERNATIONAL AGENCY FOR RESEARCH ON CANCER - IARC. Some non-heterocyclic polycyclic aromatic hydrocarbons and some related exposures: monographs on the evaluation of carcinogenic risks in humans. Lyon: IARC, 2010

JANOSZKA, B. HPLC-fluorescence analysis of polycyclic aromatic hydrocarbons (PAHs) in pork meat and its gravy fried without additives and in the presence of onion and garlic. Food Chemistry, v. 126, n. 3, p. 1344-1353, 2011. http://dx.doi. org/10.1016/j.foodchem.2010.11.097.

KATAYAMA, A.; BHULA, R.; BURNS, G. R.; CARAZO, E.; FELSOT, A.; HAMILTON, D.; HARRIS, C.; KIM, Y. H.; KLETER, G.; KOEDEL, W.; LINDERS, J.; PEIJNENBURG, J. G.; SABLJIC, A.; STEPHENSON, R. G.; RACKE, D. K.; RUBIN, B.; TANAKA, K.; UNSWORTH, J.; WAUCHOPE, R. D. Bioavailability of xenobiotics in the soil environment. Reviews of Environmental Contamination and Toxicology, v. 203, p. 1-86, 2010. PMid:19957116. http://dx. doi. org/10.1007/978-1-4419-1352-4_1.

KEHLET, U.; MEINERT, L.; AASLYNG, M. D. The effects of addition of antioxidants, pan-frying temperature and microwave heating on formation of heterocyclic aromatic amines in pork products. In: INTERNATIONAL CONGRESS OF MEAT SCIENCE AND TECHNOLOGY, 57., 2011, Ghent, Belgium. Proceedings... Ireland: ICOMST, 2011.

KUMARI, R.; CHATURVEDI, P.; ANSARI, N. G.; MURTHY, R. C.; PATEL, D. K. Optimization and validation of an extraction method for the analysis of polycyclic aromatic hydrocarbons in chocolate candies. Journal of Food Science, v. 71, n. 1, p. 34, 2012. http://dx.doi.org/10.1111/j.1750-3841.2011.02488.x.

LARSSON, B. Polycyclic aromatic hydrocarbons in Swedish foods: aspects on analysis, occurrence and intake. Tese (Doutorado)-Swedish University of Agricultural Sciences, Sweden, 1986.

LARSSON, B. K.; ERIKSON, A. T.; CERVENKA, M. Polycyclic aromatic hydrocarbons in crude and deodorized vegetable oils. Journal of the American Oil Chemists' Society, v. 64, n. 3, p. 365-370, 1987. http://dx.doi.org/10.1007/BF02549296.

LIJINSKY, W.; ROSS, A. E. Production of carcinogenic polynuclear hydrocarbons in the cooking of food. Food and Cosmetics Toxicology, v. 5, n. 3, p. 343-347, 1967. PMid:6054351. http:// dx.doi.org/10.1016/S0015-6264(67)83061-X.

LIMA, R. F.; DIONELLO, R. G.; PERALBA, M. C.; BARRIONUEVO, S.; RADUNZ, L. L.; REICHERT JÚNIOR, F. W. PAHs in corn grains submitted to drying with firewood. Food Chemistry, v. 215, p. 165-170, 2017. PMid:27542463. http://dx.doi.org/10.1016/j. foodchem.2016.07.164.

LIN, D.; TU, Y.; ZHU, L. Concentrations and health risk of polycyclic aromatic hydrocarbons in tea. Food and Chemical Toxicology, v. 43, n. 1, p. 41-48, 2005. PMid:15582194. http:// dx.doi.org/10.1016/j.fct.2004.08.010.

LODOVICI, M.; DOLARA, P.; CASALINI, C.; CIAPPELLANO, S.; TESTOLIN, G. Polycyclic aromatic hydrocarbon contamination in the Italian diet. Food Additives and Contaminants, v. 12, n. 5, p. 703-713, 1995. PMid:8522036. http://dx.doi. org/10.1080/02652039509374360.

LOPES, W. A.; ANDRADE, J. B. Fontes, formação, reatividade e quantificação de hidrocarbonetos policíclicos aromáticos (HPA) na atmosfera. Química Nova, v. 19, p. 497-516, 1996.

MCGRATH, T. E.; WOOTEN, J. B.; GEOFFREY CHAN, W.; HAJALIGOL, M. R. Formation of polycyclic aromatic hydrocarbons from tobacco: the link between low temperature residual solid (char) and PAH formation. Food and Chemical Toxicology, v. 45, n. 6, p. 1039-1050, 2007. PMid:17303297. http://dx.doi. org/10.1016/j.fct.2006.12.010.

MEADOR, J. P.; STEIN, J. E.; REICHERT, W. L.; VARANASI, U. Bioaccumulation of polycyclic aromatic hydrocarbons by marine organisms. Reviews of Environmental Contamination and Toxicology, v. 143, p. 79-165, 1995. PMid:7501868. http://dx.doi. org/10.1007/978-1-4612-2542-3_4.

MORET, S.; CONTE, L. S. Polycyclic aromatic hydrocarbons in edible fats and oils: occurrence and analytical methods. Journal of Chromatography A, v. 882, p. 245-248, 2000.

NACCARI, C.; CRISTANI, M.; GIOFRÈ, F.; FERRANTE, M.; SIRACUSA, L.; TROMBETTA, D. PAHs concentration in heattreated milk samples. Food Research International, v. 44, n. 3 , p. 716-724, 2011. http://dx.doi.org/10.1016/j.foodres.2010.12.029.

NACCARI, C.; CRISTANI, M.; GIOFRÈ, F.; LICATA, P.; TROMBETTA, $D$. Levels of benzo(a) pyrene and benzo(a)anthracene in smoked "provola" cheese from Calabria (Italy). Food Additives \& Contaminants B, v. 1, n. 1, p. 78-84, 2008.

NACCARI, F.; MARTINO, D.; TROMBETTA, D.; CRISTANI, M.; LICATA, P.; NACCARI, C. et al. Trace elements in bovine milk from dairy farms in Sicily. Italian Journal of Food Science, $v$. 18, p. 22-26, 2006.

NAKAYAMA, T.; YAMADA, M.; OSAWA, T.; KAWAKISHI, S. Suppression of active oxygen-induced cytotoxicity of flavonoids. Biochemical Pharmacology, v. 45, n. 1, p. 265-267, 1993. PMid:8381006. http://dx.doi.org/10.1016/0006-2952(93)90402-I.

NURSTEN, $H$. The Maillard reaction, chemistry, biochemistry and implications. Reading: The University of Reading, The Loyal Society of Chemistry, 2005. p. 151-152. 
Presença de hidrocarbonetos policíclicos aromáticos em produtos alimentícios e a sua relação com o método de cocção e a natureza do alimento Paz, A. P. S. et al.

PHILLIPS, D. H. Polycyclic aromatic hydrocarbons in the diet. Mutation Research, v. 443, n. 1-2, p. 139-147, 1999. PMid:10415437. http://dx.doi.org/10.1016/S1383-5742(99)00016-2.

PUPIN, A. M.; TOLEDO, M. C. F. Benzo(a)pyrene in Brazilian vegetable oils. Food Add Contam A., v. 13, n. 6, p. 639-645, 1996. http://dx.doi.org/10.1080/02652039609374449.

PURCARO, G.; NAVAS, J. A.; GUARDIOLA, F.; CONTE, L. S.; MORET, S. Polycyclic aromatic hydrocarbons in frying oils and snacks. Journal of Food Protection, v. 69, n. 1, p. 199-204, 2006. PMid:16416919. http://dx.doi.org/10.4315/0362-028X-69.1.199.

REY-SALGUEIRO, L.; GARCÍA-FALCÓN, M. S.; MARTÍNEZCARBALLO, E.; SIMAL-GÁNDARA, J. Effects of toasting procedures on the levels of polycyclic aromatic hydrocarbons in toasted bread. Food Chemistry, v. 108, n. 2, p. 607-615, 2008. http:// dx.doi.org/10.1016/j.foodchem.2007.11.026

REY-SALGUEIRO, L.; MARTÍNEZ-CARBALLO, E.; GARCÍAFALCÓN, M. S.; GONZÁLEZ-BARREIRO, C.; SIMAL-GÁNDARA, J. Occurrence of Polycyclic Aromatic Hydrocarbons and their hydroxylated metabolites in infant foods. Food Chemistry, v. 115, n. 3, p. 814-819, 2009. http://dx.doi.org/10.1016/j. foodchem.2008.12.095.

RODRIGUES, E.; CASTAGNA, A. A.; DIAS, M. T.; ARONOVICH, M. Qualidade do leite e derivados: processos, processamento tecnológico e índices. Niterói: PESAGRO-RIO, 2013. Disponível em: <http://www.pesagro.rj.gov.br/downloads/riorural/37_Qualidade_ Leite_Derivados.pdf>. Acesso em: 1 out. 2014.

ROSE, M.; HOLLAND, J.; DOWDING, A.; PETCH, S. R.; WHITE, S.; FERNANDES, A.; MORTIMER, D. Investigation into the formation of $\mathrm{PAH}$ in foods prepared in the home to determine the effects of frying, grilling, barbecuing, toasting and roasting. Food and Chemical Toxicology, v. 78, p. 1-9, 2015. PMid:25633345. http:// dx.doi.org/10.1016/j.fct.2014.12.018.

ROSEIRO, L. C.; GOMES, A.; SANTOS, C. Influence of processing in the prevalence of polycyclic aromatic hydrocarbons in a Portuguese traditional meat product. Food and Chemical Toxicology, v. 49, n. 6, p. 1340-1345, 2011. PMid:21419819. http://dx.doi.org/10.1016/j.fct.2011.03.017.

RYCHEN, G.; CREPINEAU-DUCOULOMBIER, C.; GROVA, N.; JURJANZ, S.; FEIDT, C. Modalites et risques de transfert des polluants organiques persistants vers le lait. INRA Productions Animales, v. 18, p. 355-366, 2005.

RYCHEN, G.; JURJANZ, S.; TOUSSAINT, H.; FEIDT, C. Dairy ruminant exposure to persistent organic pollutants and excretion to milk. Animal, v. 2, n. 2, p. 312-323, 2008. PMid:22445026. http://dx.doi.org/10.1017/S1751731107001139.

SCHAUM, J.; SCHUDA, L.; WU, C.; SEARS, R.; FERRARIO, J.; ANDREWS, K. A national survey of persistent, bioaccumulative and toxic (PBT) pollutants in the United States milk supply. Journal of Exposure Analysis and Environmental Epidemiology, v. 13, n.
3, p. 177-186, 2003. PMid:12743612. http://dx.doi.org/10.1038/ sj.jea.7500269.

SHIMADA, T. Xenobiotic-metabolizing enzymes involved in the activation and detoxification of carcinogenic polycyclic aromatic hydrocarbons. Drug Metabolism and Pharmacokinetics, v. 24, n. 4, p. 257-276, 2006. PMid:16946553. http://dx.doi.org/10.2133/ dmpk.21.257.

SHON, M. Y.; CHOI, S. D.; KAHNG, G. G.; NAM, S. H.; SUNG, N. J. Antimutagenic, antioxidant and free radical scavenging activity of ethyl acetate extracts from white, yellow and red onions. Food and Chemical Toxicology, v. 42, n. 4, p. 659-666, 2004. PMid:15019191. http://dx.doi.org/10.1016/j.fct.2003.12.002.

SILVA, F. S.; CRISTALE, J.; RIBEIRO, M. L.; MARCHI, M. R. R. Polycyclic aromatic hydrocarbons (PAHs) in raw cane sugar (rapadura) in Brazil. Journal of Food Composition and Analysis, v. 24, n. 3, p. 346-350, 2011. http://dx.doi.org/10.1016/j. jfca.2010.08.012.

SILVA, R. B.; WOLQUIND, C. S.; SILVA, F. S.; PORTO, A. G.; SILVA, F. T. C. Aplicação da produção mais limpa no processo de clarificação do caldo de cana para produção de açúcar. In: ENCONTRO NACIONAL DE ENGENHARIA DE PRODUÇÃO, 28., 2008, Rio de Janeiro. Anais... Rio de Janeiro: ABEPRO, 2008.

SIMONEIT, B. R. T. Biomass burning a review of organic tracers for smoke from incomplete combustion. Applied Geochemistry, v. 17, n. 3, p. 129-162, 2002. http://dx.doi.org/10.1016/S08832927(01)00061-0.

SINGH, L.; VARSHNEY, J. G.; AGARWAL, T. Polycyclic aromatic hydrocarbons' formation and occurrence in processed food. Food Chemistry, v. 199, p. 768-781, 2016. PMid:26776034. http://dx.doi.org/10.1016/j.foodchem.2015.12.074.

SMITH, J. S.; AMERI, F.; GADGIL, P. Effect of marinades on the formation of heterocyclic amines in grilled beef steaks. Journal of Food Science, v. 73, n. 6, p. 100-105, 2008. http://dx.doi. org/10.1111/j.1750-3841.2008.00856.x.

SPISSO, B. F.; NÓBREGA, A. W.; MARQUES, M. A. Resíduos e contaminantes químicos em alimentos de origem animal no Brasil: histórico, legislação e atuação da vigilância sanitária e demais sistemas regulatórios. Ciência \& Saúde Coletiva, v. 14, n. 6, p. 2091-2106, 2009. PMid:20069177. http://dx.doi. org/10.1590/S1413-81232009000600016.

STAVRIC, B.; KLASSEN, R. Dietary effects on the uptake of benzo[a]pyrene. Food and Chemical Toxicology, v. 32, n. 8 , p. 727-734, 1994. PMid:8070737. http://dx.doi.org/10.1016/ S0278-6915(09)80005-7.

TARANTINI, A.; MAITRE, A.; LEFEBVRE, E.; MARQUES, M.; RAJHI, A.; DOUKI, T. Polycyclic aromatic hydrocarbons in binary mixtures modulate the efficiency of benzo[a]pyrene to form DNA adducts in human cells. Toxicology, v. 279, n. 1-3, p. 36-44, 2011. PMid:20849910. http://dx.doi.org/10.1016/j.tox.2010.09.002. 
Presença de hidrocarbonetos policíclicos aromáticos em produtos alimentícios e a sua relação com o método de cocção e a natureza do alimento Paz, A. P. S. et al.

TEIXEIRA, V. H.; CASAL, S.; OLIVEIRA, M. B. P. P. PAHs content in sunflower, soybean and virgin olive oils: evaluation in commercial samples and during the refining process. Food Chemistry, v. 104, n. 1, p. 106-112, 2007. http://dx.doi.org/10.1016/j. foodchem.2006.11.007.

TFOUNI, S. A. V.; SOUZA, N. G.; NETO, M. B.; LOREDO, I. S. D.; LEME, F. M.; FURLANI, R. P. Z. Polycyclic aromatic hydrocarbons (PAHs) in sugarcane juice. Food Chemistry, v. 116, n. 1, p. 391-394, 2009. http://dx.doi.org/10.1016/j.foodchem.2009.02.040.

VIEGAS, O.; NOVO, P.; PINTO, E.; PINHO, O.; FERREIRA, I. M. Effect of charcoal types and grilling conditions on formation of heterocyclic aromatic amines (HAs) and polycyclic aromatic hydrocarbons (PAHs) in grilled muscle foods. Food and Chemical Toxicology, v. 50, n. 6, p. 2128-2134, 2012. PMid:22459130. http://dx.doi.org/10.1016/j.fct.2012.03.051.

WEIS, B.; CHAIM, N. A.; BELIK, W. Manual de gestão eficiente da merenda escolar. São Paulo: Margraf, 2004. Disponível em: <http://www.adital.org.br/fomezero/images/merenda.pdf>. Acesso em: 1 out. 2014.

WORLD HEALTH ORGANIZATION - WHO. Summary and conclusions of the sixty-fourth meeting of the joint FAO/WHO Expert Committee on Food Additives. Rome: WHO, 2005.

WRETLING, S.; ERIKSSON, A.; ESKHULT, G. A.; LARSSON, B. Polycyclic aromatic hydrocarbons (PAHs) in Swedish smoked meat and fish. Journal of Food Composition and Analysis, v. 23, n. 3, p. 264-272, 2010. http://dx.doi.org/10.1016/j.jfca.2009.10.003.

XIA, Z.; DUAN, X.; QIU, W.; LIU, D.; WANG, B.; TAO, S.; JIANG, Q.; LU, B.; SONG, Y.; HU, X. Health risk assessment on dietary exposure to polycyclic aromatic hydrocarbons (PAHs) in Taiyuan, China. The Science of the Total Environment, v. 408, n. 22, p. 5331-5337, 2010. PMid:20800879. http://dx.doi.org/10.1016/j. scitotenv.2010.08.008.

ZAMPERLINI, G. C. M.; SANTIAGO-SILVA, M.; VILEGAS, W. Solidphase extraction of sugar cane soot extract for analysis by gas chromatography with flame ionization and mass spectrometric detection. Journal of Chromatography A, v. 889, n. 1-2, p. 281-286, 2000. PMid:10985559. http://dx.doi.org/10.1016/ S0021-9673(00)00291-0.

ZHANG, H.; XUE, M.; DAI, Z. Determination of polycyclic aromatic hydrocarbons in aquatic products by HPLC-fluorescence. Journal of Food Composition and Analysis, v. 23, n. 5, p. 469-474, 2010. http://dx.doi.org/10.1016/j.jfca.2009.12.016.

ZHANG, Y.; TAO, S. Global atmospheric emission inventory of polycyclic aromatic hydrocarbons (PAHs) for 2004. Atmospheric Environment, v. 43, n. 4, p. 812-819, 2009. http://dx.doi. org/10.1016/j.atmosenv.2008.10.050.

ZHANG, Y.; TAO, S.; SHEN, H.; MA, J. Inhalation exposure to ambient polycyclic aromatic hydrocarbons and lung cancer risk of Chinese population. Proceedings of the National Academy of Sciences of the United States of America, v. 106, n. 50, p. 21063-21067, 2009. PMid:19995969. http://dx.doi.org/10.1073/ pnas.0905756106.

ZIEGENHALS, K.; SPEER, K.; JIRA, W. Polycyclic aromatic hydrocarbons $(\mathrm{PAH})$ in chocolate on the German market. Journal für Verbraucherschutz und Lebensmittelsicherheit, v. 4, n. 2, p. 128-135, 2009. http://dx.doi.org/10.1007/s00003-009-0478-1. 